\title{
BMJ Open Study protocol of a phase 1 clinical trial establishing the safety of intrapleural administration of liposomal curcumin: curcumin as a palliative treatment for malignant pleural effusion (IPAL-MPE)
}

To cite: Hocking AJ, Farrall AL, Newhouse S, et al. Study protocol of a phase 1 clinical trial establishing the safety of intrapleural administration of liposomal curcumin: curcumin as a palliative treatment for malignant pleural effusion (IPAL-MPE). BMJ Open 2021;11:e047075. doi:10.1136/ bmjopen-2020-047075

- Prepublication history and additional material for this paper is available online. To view these files, please visit the journal online (http://dx.doi.org/10. 1136/bmjopen-2020-047075).

AJH and ALF are joint first authors.

Received 20 November 2020 Revised 19 February 2021 Accepted 24 February 2021

Check for updates

(C) Author(s) (or their employer(s)) 2021. Re-use permitted under CC BY-NC. No commercial re-use. See rights and permissions. Published by BMJ.

For numbered affiliations see end of article.

Correspondence to Dr Ashleigh Jean Hocking; ash.hocking@flinders.edu.au

\section{ABSTRACT}

Introduction This is a phase 1, open-label, singlecentre, uncontrolled, dose-escalation study to evaluate the feasibility, tolerability and pharmacokinetic profiles of a single dose of liposomal curcumin, administered via an existing tunnelled indwelling pleural catheter (TIPC) directly to the tumour site in individuals with diagnoses of malignant pleural effusion. Primarily, we aim to determine a maximum tolerated dose of liposomal curcumin administered via this method.

Methods and analysis We will use a $3+3$ expanded cohort for predefined dose-escalation levels or until a predefined number of dose-limiting toxicities are reached. Participants will be administered a single dose of liposomal curcumin (LipoCurc, SignPath Pharma) via their existing TIPC as a sequential enrolling case series with the following dose cohorts: 100, 200 and $300 \mathrm{mg} / \mathrm{m}^{2}$. Primary endpoints are determination of the maximum tolerated dose within the predetermined dose range, and determination of the feasibility of intrapleural administration of liposomal curcumin via an existing TIPC. Secondary endpoints include determination of the safety and tolerability of intrapleural administration of liposomal curcumin, median overall survival, effects on quality of life and on feelings of breathlessness, and the pharmacokinetics and concentrations of curcumin from the plasma and the pleural fluid. Important inclusion criteria include age $\geq 18$ years, an existing TIPC, a pleural biopsy or pleural fluid cytology-proven diagnosis of malignant pleural effusion and for whom no antitumour therapy of proven benefit is available or has been previously declined, eastern cooperative group performance status $<2$.

Ethics and dissemination The study protocol has been approved by the Southern Adelaide Local Health Network Human Research Ethics Committee (HREC) (approval number: HREC/20/SAC/11). Study results will be published in peer-reviewed journals, and presented at conferences, in field of medical oncology and respiratory medicine.

Trial registration number ACTRN12620001216909. Protocol version number V.1.0.

\section{Strengths and limitations of this study}

To our knowledge, this is the first study in which curcumin of any formulation will be delivered directly into the pleural cavity in humans.

- Due to the pharmacokinetic nature of curcumin namely poor bioavailability and rapid degradation, direct intrapleural delivery of liposome encapsulated curcumin may better provide clinically relevant concentrations of curcumin to tumours at this site.

- The study design does not allow for the evaluation of the safety of multiple doses of liposomal curcumin. Multiple doses may be required to assess for sustained symptomatic relief, or a potential antineoplastic effect, in participants in subsequent phase II studies.

\section{INTRODUCTION}

Malignant pleural effusion

A malignant pleural effusion (MPE) is the accumulation of fluid in the pleural cavity as a result of primary or secondary pleural malignancy. The most prevalent causes of MPE are late-stage breast and lung cancers that have metastasised to the pleural or mediastinal lymph nodes. ${ }^{1}$ Patients presenting with an MPE have a poor prognosis, with median survival times between 1 and 12 months depending of the type and stage of the underlying malignancy. ${ }^{2-4}$ Controlling the pleural effusion is an integral part of managing patient symptoms as an MPE causes significant discomfort, breathing difficulties and commonly recurs after the initial therapeutic drainage. ${ }^{1}$ Recurrent pleural effusion can be managed by either talc pleurodesis or insertion of a tunnelled indwelling pleural catheter (TIPC) ${ }^{5-7}$ At present, there is a lack of evidence supporting the use of anticancer 
therapies as an alternative to MPE drainage, ${ }^{6}$ and so the pursuit of new possible treatments, or adjuvant therapy options and approaches for MPE, from early intervention all the way through to palliative stages, remains an open challenge.

\section{Curcumin and cancer}

Curcumin is a polyphenol derived from the spice turmeric, which can modulate numerous pathways involved in carcinogenesis, including those controlling inflammation, cell cycle progression, angiogenesis and cell survival. Curcumin also has the potential to help to reduce pleural fluid production since it can reduce numerous factors involved in fluid accumulation including vascular endothelial growth factor-A, interlukin-6, signal transducer and activator of transcription 3, sphingosine phosphate, indolamine 2,3 dioxygenase and tumour necrosis factoralpha. ${ }^{18-10}$ The translation of these anticancer effects into a clinical setting has been hampered because of curcumin's low solubility, instability at physiological $\mathrm{pH}$, low bioavailability and rapid molecular transformation and degradation in the blood. ${ }^{11}$ In order to combat this, a variety of curcumin formulations and drug delivery systems have been developed to improve its stability, solubility and bioavailability in vivo including lipid-based vehicles such as curcumin-loaded micelles and liposomes. ${ }^{12}$

\section{Liposomal curcumin}

Liposomes are phospholipid vesicles that are used as delivery systems for drugs to reduce early degradation and improve stability, biodistribution and cellular uptake. ${ }^{13} 14$ A pharmaceutical-grade, liposome-encapsulated synthetic curcumin, called 'LipoCurc' is currently the only liposomal curcumin formulation that has been administered intravenously in humans-in both healthy humans and advanced cancer patients. ${ }^{15} 16$ In patients with cancer, liposomal curcumin $\left(100-300 \mathrm{mg} / \mathrm{m}^{2}\right)$ was administered intravenously over a period of $6-8$ hours, weekly for 8 weeks or until the disease progressed or intolerable toxicity was observed. The authors observed a significant increase in haematological adverse events (AEs) in patients receiving the $300 \mathrm{mg} / \mathrm{m}^{2}$ treatment, including one case of dose-limiting haemolysis. A significant tumour marker response and temporary clinical benefit were observed in two patients after receiving $300 \mathrm{mg} / \mathrm{m}^{2}$ of liposomal curcumin. ${ }^{16}$ However, following intravenous drug delivery, a drug may be unevenly redistributed to organs tissues, and fluids ${ }^{17}$ and thus only a small proportion of the administered dose may reach the pleural cavity.

\section{Rationale for phase 1 trial of intrapleural administration of liposomal curcumin}

The position of the tumour cells adjacent to the pleural cavity provides a unique opportunity to administer therapeutics directly to the tumour site. Intrapleural liposomal curcumin therapy offers several potential advantages over intravenous therapy in patients with primary and secondary malignancies of the pleura. Most importantly, it allows the delivery of a high dose of curcumin directly to the site of the tumour and, in reality; this may be the only method to deliver clinically relevant concentrations of curcumin to the pleural cavity while minimising potential systemic toxicity. Liposome encapsulated chemotherapeutic drugs have been administered into the pleural cavity in both phase I and phase II clinical trials in humans with malignant pleural mesothelioma before to some effect. ${ }^{18} 19$ Therapeutic delivery via an existing TIPC means patients could be given tumour-site targeted therapies while also avoiding any additional instrumentation of the pleura, therefore, reducing procedural risk.

\section{Justification of intrapleural curcumin dose and escalation schedule}

We have elected to test the safety of three predetermined single escalating dose levels of liposomal curcumin (LipoCurc). Dosage levels will be body surface area adjusted to $100 \mathrm{mg} / \mathrm{m}^{2}, 200 \mathrm{mg} / \mathrm{m}^{2}$ and $300 \mathrm{mg} / \mathrm{m}^{2}$. These dose concentrations are below the previously established safe systemic dosage levels of liposomal curcumin determined in healthy adults (up to $400 \mathrm{mg} / \mathrm{m}^{2}$ ), ${ }^{15}$ and in line with intravenous dosage levels assessing liposomal curcumin safety and efficacy for the treatment of metastatic cancers in a phase I clinical trial (maximum tolerated dose (MTD) $\left.300 \mathrm{mg} / \mathrm{m}^{2}\right) .{ }^{16}$ Body surface area adjusted doses have been used in clinical trials that assessed the safety and efficacy of drugs administered into the pleural and peritoneal cavities in humans, including liposomeentrapped antineoplastic therapies. ${ }^{18-21}$ Our preclinical studies in healthy rats also demonstrated low systemic curcumin absorption following intrapleural administration of liposomal curcumin (LipoCurc). Furthermore, our study showed that intrapleural doses of liposomal curcumin equivalent to $300 \mathrm{mg} / \mathrm{m}^{2}$ in humans did not cause any pleural pathology. ${ }^{22}$ The MTD for liposomeentrapped chemotherapy delivered to the pleural cavity in humans was $50 \%$ higher than the MTD after intravenous administration of the same dose. ${ }^{19}$ In light of these studies, we do not anticipate that the MTD will be reached at the highest planned dosage level.

\section{METHODS}

We used the Standard Protocol Items: Recommendations for Interventional Trials checklist when preparing our protocol for publication. ${ }^{23}$

\section{Aim}

This phase I study aims to assess the safety and feasibility of a single dose of intrapleural liposomal curcumin (LipoCurc) in patients with MPE, administered via their existing TIPC.

\section{Primary objectives}

To determine:

1. If the MTD of intrapleural liposomal curcumin is reached within the predetermined escalating dose range in people with an advanced MPE. 
2. The feasibility of intrapleural administration of liposomal curcumin via an existing TIPC.

\section{Secondary objectives}

1. To evaluate safety and tolerability by determining:

a. The rates of toxicities based on the National Cancer Institute Common Terminology Criteria for Adverse Events V.5.0 (NCI CTCAE V.5.0).

b. The effects of the study intervention on quality of life based on the average scores as assessed by the European Organisation for Research and Treatment of Cancer Quality of Life Questionnaire (EORTC-QLQ) ${ }^{24}$

c. The effects of the study intervention on feeling of breathlessness, based on the average scores as assessed by the Visual Analogue Scale for Breathlessness survey (VASB survey).

d. Median overall survival.

2. To evaluate the pharmacokinetics of curcumin in all participants following administration of a single dose of liposomal curcumin in to the intrapleural cavity by measuring the concentrations of curcumin and its metabolites in the plasma and, where possible, pleural fluid.

3. To evaluate any evidence of anti-tumour activity as indicated by clinical outcomes, in conjunction with cellular and molecular analysis of participant's biospecimens collected for diagnostic purposes and surplus to diagnostic needs.

\section{STUDY DESIGN}

Open label, single-centre, phase 1 trial modelled on a standard 3+3 dose-escalation design.

\section{PARTICIPANT POPULATION Target population}

People in palliative care with a cytopathologically or histopathologically proven diagnosis of MPE, and where the insertion of a TIPC is indicated by the treating clinician.

\section{Inclusion criteria}

1. Age $\geq 18$ years.

2. An existing diagnosis of MPE proven by either:

a. Pleural biopsy.

b. Pleural fluid cytology in conjunction with typical radiological and clinical findings.

3. Individuals who have:

a. Failed to respond to approved systemic therapies (chemotherapy, immune therapy or molecular targeted therapies), or who have progressive cancers following initial response to these therapies, and for whom no antitumour therapy of proven benefit is available at study enrolment.

b. Who have declined systemic therapies.

c. Are deemed not suitable for systemic therapies after consultation with a medical oncologist.
4. Recurrent symptomatic pleural effusion where insertion of a TIPC is clinically indicated.

5. An Eastern Cooperative Group performance status of 0-2.

6. Able to give signed informed consent.

\section{Exclusion criteria}

1. Any comorbidities or conditions that the investigator considers the patient should not participate in the study include:

a. Evidence of active hepatitis.

b. People with a diagnosis of lymphoma or haematological cancer.

c. People with a history of haemolytic anaemia.

d. People with unresolved toxicities from prior systemic anticancer therapy.

e. People with an unstable cardiac condition as determined by the study investigators.

2. Women who are pregnant and/or breast feeding, and/or of childbearing age not taking contraceptive measures to avoid pregnancy.

3. People with mental impairment, or an unstable medical condition other than cancer that may interfere with their ability to provide informed consent or ability to cooperate and participate in the study.

4. People whose primary language is not English.

5. People who are taking anticoagulation medication, including warfarin, clexane and/or direct oral anticoagulants.

\section{Participant screening and registration}

All inclusion and exclusion criteria are assessed as a part of standard practice of care and/or before TIPC insertion. With no exceptions, prospective participants that meet all the inclusion criteria, and none of the exclusion criteria, will be eligible for participation and will be offered the opportunity to take part in the clinical trial. Written informed consent must be signed and dated by the participant, and the investigator, prior to any studyspecific procedures or activities.

\section{PARTICIPANT ENROLMENT AND TREATMENT PLAN}

Participants will enter the trial and be treated as a consecutively enrolling case series as outlined in table 1 .

\section{Insertion of TIPC}

The insertion of a TIPC will occur at least 1 week before the administration of the liposomal curcumin, to allow for monitoring and treatment of any potential postprocedure complications. Patients will remain in the hospital for 24-48 hours after TIPC insertion (as per our local protocol) and medical/nursing staff will monitor patients until deemed medically fit to be discharged by the clinician. Pleural fluid drainage will be performed via a vacuum bottle system (PleurX Drainage System). The volume and frequency of drainage will be determined and managed by the clinical team. Drained pleural fluid 


\begin{tabular}{llll} 
Table 1 & Dosing of intrapleural Lipocurc based on 3+3 design \\
Cohort & $\begin{array}{l}\text { No of } \\
\text { patients }\end{array}$ & $\begin{array}{l}\text { Lipocurc dose } \\
\text { given on day } 1\end{array}$ & Escalation \\
\hline 1 & 3 & $100 \mathrm{mg} / \mathrm{m}^{2}$ & $\begin{array}{l}\text { If no dose-limiting toxicity is seen after a minimum of three patients have completed } \\
\text { treatment in cohort 1, enrolment will commence for cohort } 2 \text { participants. }\end{array}$ \\
2 & 3 & $\begin{array}{l}200 \mathrm{mg} / \mathrm{m}^{2} \\
\text { If no dose-limiting toxicity is seen after a minimum of three patients have completed } \\
\text { treatment in cohort 2, enrolment will commence for cohort } 3 \text { participants. }\end{array}$ \\
3 & $300 \mathrm{mg} / \mathrm{m}^{2}$ & $\begin{array}{l}\text { If no dose-limiting toxicity is seen after three patients have completed treatment in } \\
\text { cohort 3, there will be no further dose escalation. This cohort will be expanded to six } \\
\text { patients if maximum tolerated dose has not been reached. }\end{array}$
\end{tabular}

obtained from the effusion will be collected, and cells separated by centrifugation, for pathology monitoring and testing. Baseline studies will be performed after TIPC insertion, as outlined in table 2. Participants' renal function will be estimated by estimated glomerular filtration rate (eGFR) using their body weight and urea and electrolytes prior to the CT scan to determine if a CT with intravenous contrast can be performed. Participants with an eGFR $>30 \mathrm{~mL} / \mathrm{min} / 1.73 \mathrm{~m}^{2}$ may have intravenous contrast in accordance with the 2011 Iodinated Contrast Guidelines from the Royal Australia and New Zealand College of Radiologists. Participants with an eGFR $<30 \mathrm{~mL} / \mathrm{min} / 1.73 \mathrm{~m}^{2}$ will not have intravenous contrast for CT scans.

\section{Intrapleural administration of liposomal curcumin via an existing TIPC}

A week after the insertion of the TIPC, participants will attend the clinic as an outpatient for suture removal and vacuum bottle drainage. Baseline studies will be taken, as outlined in table 2. Participants will then be admitted as a hospital inpatient for the intrapleural liposomal curcumin administration. Before intrapleural administration of the liposomal curcumin, pleural fluid will be drained via the TIPC using the vacuum drainage bottle system. Liposomal curcumin will be prepared under aseptic conditions by the treating medical team and administered at room temperature through the TIPC via an adaptor port (PleurX catheter Access Kit). Drug delivery will take 10-15 min and will be followed by sequential $10 \mathrm{~mL}$ flushes of room temperature sterile $0.9 \%$ saline until the TIPC is visibly clear of the yellow-coloured liposomal curcumin solution. Participants will be admitted as an inpatient for 48 hours to allow time to monitor for any potential serious adverse events (SAEs). The participant will be monitored by medical and nursing staff, with observations of blood pressure, pulse, oxygen saturation and temperature every $15 \mathrm{~min}$ for 1 hour, and then every hour for 4 hours, then

Table 2 Scheduled visits and associated procedures required during the phase 1 study assessing the safety and feasibility of intrapleural administration of Lipocurc via an existing TIPC

\begin{tabular}{|c|c|c|c|c|c|c|c|c|}
\hline Week & Location & $\begin{array}{l}\text { Blood tests, } \\
\text { including, } \\
\text { circulating } \\
\text { curcumin }\end{array}$ & Vitals & $\begin{array}{l}\text { Pleural } \\
\text { effusion } \\
\text { fluid } \\
\text { collection }\end{array}$ & $\begin{array}{l}\text { VASB } \\
\text { survey }\end{array}$ & $\begin{array}{l}\text { EORTC } \\
\text { QLQ-C30 }\end{array}$ & Chest X-ray & Chest CT \\
\hline $\begin{array}{l}\text { Week } 0 \\
\text { IPA-Lipocurc }\end{array}$ & $\begin{array}{l}\text { Clinic+In-patient } \\
48 \text { hours }\end{array}$ & $\begin{array}{l}\checkmark \\
\text { Pre-IPA,+2, } \\
24 \text { and } \\
48 \text { hours } \\
\text { Post-IPA }\end{array}$ & $\checkmark$ & $\begin{array}{l}\checkmark \\
\text { Pre-IPA }\end{array}$ & $\begin{array}{l}\checkmark \\
2,24, \\
48 \text { hours } \\
\text { Post-IPA }\end{array}$ & $\begin{array}{l}\checkmark \\
2,24, \\
48 \text { hours } \\
\text { Post-IPA }\end{array}$ & $\begin{array}{l}\checkmark \\
48 \text { hours } \\
\text { Post-IPA }\end{array}$ & $\begin{array}{l}\checkmark \\
\text { Pre-IPA }\end{array}$ \\
\hline Week 1 & Clinic & $\checkmark$ & $\checkmark$ & If possible & $\checkmark$ & $\checkmark$ & $\checkmark$ & $x$ \\
\hline Week 4 & Clinic $^{*}$ & $\checkmark$ & $\boldsymbol{J}^{*}$ & If possible & $\checkmark$ & $\checkmark$ & $\checkmark$ & $x$ \\
\hline
\end{tabular}

*Activities and procedures required for routinestandard of care for TIPC insertion and clinical care. Blood tests include, FBC, UEC, LFT, CRP; vitals monitoring includes, bloodpressure, pulse, oxygen $\left(\mathrm{O}_{2}\right)$ saturation.

CRP, C reactive protein; EORTC QLQ-C30, European Organisation for Research andTreatment of Cancer Quality of Life Questionaire-C30; FBC, full blood count; IPA-Lipocurc, Intrapleural administration of Lipocurc; LFT, liver function tests; TIPC, tunnelled indwelling pleural catheter; UEC, urea and electrolytes; VASB, Visual Analogue Scale for Breathlessnes. 
at 4 hourly intervals up to 48 hours in total. Additional postprocedural assessments will be performed according to the schedule in table 2.

Any adverse reactions will be evaluated by the NCI CTC and treated appropriately at the discretion of the respiration physician. On discharge, participants will be referred to the Royal District Nursing Service for at home drainage of the effusion.

A minimum period of at least 1 week between administration of liposomal curcumin and consecutively enrolling participants will be instituted to check for toxicities or SAEs. This time frame is in line with normal clinical practices for monitoring post-intrapleural treatments for pleural effusion. Furthermore, in preclinical studies, we did not detect any curcumin in the plasma of rats 1 week after intrapleural administration of liposomal curcumin (dose equivalent to 300 $\left.\mathrm{mg} / \mathrm{m}^{2}\right) .{ }^{22}$ Escalation to the next dosage level will be stopped if one third or greater $(\geq 33 \%)$ of cohort participants experience dose limiting toxicities. If the MTD level is reached, another three patients will be accrued at the previous dose level to assure tolerability in subsequent phase clinical trials.

Follow-up post single dose of intrapleural liposomal curcumin One week after the intrapleural administration of the single dose of liposomal curcumin, participants will be assessed as an outpatient at the Respiratory Clinic at Flinders Medical Centre and monitored as outlined in table 2. Outpatient monitoring will be repeated at $4,8,12$ and 24 weeks postintrapleural liposomal curcumin administration. Where possible, any pleural fluid drained at follow-up clinic visits will be collected for analysis.

\section{Concomitant medication and reporting}

Prohibited medications, and subject to exclusion from study participation: Anticoagulation medication; including warfarin, clexane and/or direct oral anticoagulants. There are no diet restrictions on trial participants during the study. Numerous studies have shown that orally consumed dietary curcumin—in food or as a supplement-is poorly absorbed beyond the gut mucosa, with either undetectable or negligible levels of curcumin measured in blood serum or urine. ${ }^{25-27}$ Most ingested curcumin is excreted, unchanged, in the faeces. ${ }^{28} 29$ Concomitant medications will not be recorded during the study.

\section{Patient and public involvement}

No patient or public involvement.

\section{OUTCOMES ENDPOINTS AND OTHER MEASURES}

Participants will be assessed for up to a maximum of 24 weeks post administration of the liposomal curcumin. In the event a participant dies prior to completing study participation, date of death will be recorded, and data collected prior to death will be included in study analyses.

Outcomes will be measured at weeks $0,4,8,12$ and 24 postadministration of liposomal curcumin for:

\section{Maximum tolerated dose}

MTD is defined as the highest dose level at which $\leq 33 \%$ of participants experience dose-limiting toxicity (DLT) ${ }^{30}$ DLTs will be assessed according to the Common Toxicity Evaluation Criteria according to the National Cancer Institute version 5 (NCI-CTCAE V.5.0, 2017) guidelines. ${ }^{31}$ DLTs are defined as the following AEs if they develop within 1week of receiving the trial intervention and with a reasonable suspected causal relationship to intrapleural liposomal curcumin.

\section{Dose-limiting toxici \\ Haemolysis}

1. NCI-CTC grade 2 evidence of haemolysis and $\geq 2 \mathrm{~g}$ decrease in haemoglobin, confirmation of a causal relationship to the study medication according to the investigator.

\section{Non-haematological toxicity}

1. Any NCI-CTC grade 3 or higher non-haematological toxicity, excluding nausea and vomiting which responds to antiemetic treatment and alopecia.

2. Neuro-cerebellar: intention tremor, slurred speech, nystagmus, dysmetria.

\section{Other haematological toxicity}

1. NCI-CTC grade 3 anaemia.

2. NCI-CTC grade 4 platelet toxicities.

3. NCI-CTC grade 4 granulocyte toxicity $\geq 7$ days.

4. Febrile neutropenia: defined as an absolute neutrophils count $<500 / \mathrm{mm}^{3}$ and fever either as 2 elevations of oral temperature $>38^{\circ} \mathrm{C}$ with 1-hour interval or a single oral temperature $>38.5^{\circ} \mathrm{C}$, provided that this single episode is not clearly related to other events.

AEs (worst grade according to the NCI-CTCAE V.5.0).

Any adverse reaction events will be evaluated by the NCI-CTCAE V.5.0 guidelines to classify and grade the intensity of AEs during trial participation. See the section Safety Reporting for the definition of an AE and reporting of SAEs.

\section{Effects of treatment intervention on quality of life}

Based on average scores provided from participant responses to VASB survey (online supplemental material 1) and EORTC QLQ-C30 questionnaire.

\section{Overall survival}

Overall survival is defined as the interval from on-study enrolment date, until the date of death from any cause, or cessation of study participation (the date of last known study follow-up visit alive and/or final study follow-up visit), whichever comes first.

\section{SAFETY REPORTING Definitions}

An $\mathrm{AE}$ is any medical occurrence in a trial participant administered a pharmaceutical product and which 
does not necessarily have a causal relationship with this treatment.

AEs include:

- An exacerbation, or an unexpected increase in the intensity or frequency of a pre-existing condition (other than the MPE condition under investigation), including intermittent or episodic conditions.

- Significant or unexpected worsening or exacerbation of the MPE.

- A suspected drug interaction.

- An intercurrent illness.

- Any clinically significant laboratory abnormality that requires clinical intervention or further investigation (beyond ordering a laboratory test).

- Injury or accidents.

An AE does not include:

- Anticipated day-to-day fluctuations of any pre-existing conditions, including the MPE and/or underlying malignancy.

- Signs and symptoms of the disease under study that do not represent a significant worsening or exacerbation.

- Expected progression of the MPE or underlying malignancy.

AEs with severity grades 1 or 2 as defined in the NCICTCAE V.5.0 guidelines must be reported to the principal investigator and recorded.

SAEs include:

- Any untoward medical occurrence which fulfils the definition of an AE (see above) and additionally is: fatal; life threatening; results in hospitalisation or prolongs hospital stay; disabling/incapacitating; a congenital abnormality; any important medical occurrence which the investigator regards as serious based on appropriate medical judgement.

Laboratory abnormalities identified as critical to safety evaluations that also require immediate reporting include; low red blood cell count or other indicators of haemolytic disorders.

SAEs with severity of grades 3,4 or 5 , must be immediately reported to the principal investigator within one working day of the investigator becoming aware of the event and recorded. The principal investigator must notify the study sponsor and the local Human Research Ethics Committee (HREC) as required.

Investigators must continue to monitor all participants with SAEs until the event has subsided, stabilised, otherwise explained or the participant is lost to follow-up.

All AEs, including Adverse Drug Reactions (ADRs), SAEs or Serious Adverse Drug Reactions (serious ADR), will be prospectively tabulated for the duration of the trial to inform ongoing safety and toxicity monitoring by the safety review/dose-escalation committee, annual reports to the SAC HREC and the final study report.

\section{Pregnancy}

The effects of liposomal curcumin on the unborn child and on the newborn baby are not known. Both male and female participants will be strongly advised to use effective contraception for the period of time they are actively participating in this research project, and for a period of 90 days after receiving the single dose of the test drug. As a precaution, in the event a participant becomes pregnant during study participation, they will be withdrawn from the study. Male participants who father a child while participating in the research project and/or up to 90 days after administration of the liposomal curcumin should be reported to the study investigators. The study doctor will advise on any medical attention required for the pregnant partner should this be necessary.

\section{STATISTICAL CONSIDERATIONS AND DATA ANALYSIS PLAN}

This is a phase 1 uncontrolled, open-label, dose-escalation study primarily for the assessment of safety using a standard $3+3$ dose-escalation design, ${ }^{30}$ therefore, does not require sample size justification. The dose escalation will be continued until the predefined dose level and/or the MTD is reached. Analyses will be primarily descriptive and observational to characterise DLTs, and response rates.

\section{Test drug information: LipoCurc}

The liposomal curcumin test drug, LipoCurc, will be manufactured by SignPath Pharma (USA), and tested and packaged and labelled by Polymun Scientific, Austria in compliance with Good Manufacturing Practice (GMP). Curcumin will be synthesised at under GMP conditions to 99.2\% purity. The use of LipoCurc for this phase 1 trial is conditional on its registration with the Therapeutic Goods Administration of Australia by the study sponsor, Flinders University, under the Clinical Trial Notification scheme.

\section{STUDY ADMINISTRATION}

\section{Ethics and dissemination}

This single site study was approved by the Southern Adelaide Local Health Network Human Research Ethics Committee (approval number: HREC/20/SAC/11). On study completion, a manuscript will be prepared for publication. This study will be conducted in accordance with the Integrated Addendum to International Council For Harmonisation (ICH) E6(R1): Guideline For Good Clinical Practice ICH E6(R2) (ICH Of Technical Requirements For Pharmaceuticals For Human Use (ICH), 2016), the Note for Guidance on Good Clinical Practice (CPMP/ICH/135/95) annotated with TGA comments (Therapeutic Goods Administration DSEB July 2000), and in compliance with applicable laws and regulations. The study will be performed in accordance with the Australian National Statement on Ethical Conduct in Human Research (updated 2018, Commonwealth of Australia 2007), and the National Health and Medical Research Council Australian Code for Responsible Conduct of Research (Commonwealth of Australia 2007), and the principals laid down in the Declaration of Helsinki 2008 by the World Medical Assembly. 


\section{Recruitment of participants}

Prospective participants will be recruited from the pool of patients presenting at Flinders Medical Centre, South Australia, for the management of their MPE. Whenever possible, an independent respiratory clinician working at FMC will directly approach and discuss possible recruitment to the study with preidentified (prescreened) candidates for study participation at the time that standard TIPC insertion is explained to patients. If they express and interest in the study, they will be provided with a copy of the study participant information and consent form by a member of the research team inviting them to voluntarily participate in the research study. Prospective participants will also be encouraged to discuss trial participation with others, including with their family and their own doctor, as well as with the study team prior to granting their consent. Prospective participants will have about 1-2 weeks to consider if they will grant their consent. Persons who decide to participate will meet with a study investigator to sign a consent form.

\section{Consent}

Prescreening of prospective participants will be conducted in compliance with the waiver of consent granted for this process. Each participant's voluntary decision to take part in the study will be established by written informed consent documented on the participant information and consent form which will be signed and dated by the participant and by the witnessing consenting clinician, or member of the trial research team. All participants will be provided with a signed and dated copy of their informed consent. Written informed consent must be obtained prior to any study specific procedures or activities.

\section{Confidentiality}

All data generated in this study will remain confidential in accordance with applicable privacy acts and regulations. All data will be securely stored and made only available to members of the study research team and who have signed a confidentiality agreement. Study data will be analysed and reported in a largely aggregated manner, and trial participants will be anonymous in any presentations or publications arising from this study.

\section{Protocol amendments}

Any changes and amendments to the protocol can only be made by the principal investigator. Protocol changes can only be implemented on approval by the institutional HREC.

\section{Data handling and record keeping}

Trial data required for the monitoring and analysis of the study will be recorded on the case reports forms and associated documentation of laboratory investigations and outcomes. Accuracy of the completed case report forms will be indicated by the signing by the study investigator. All study data will be maintained for a minimum of 15 years following completion of the study in accordance with the Australian Code for the Responsible Conduct of Research. $^{32}$

\section{STUDY MONITORING}

\section{Safety review/dose-escalation committee}

A safety review/dose-escalation committee will function in consultation with independent respiratory physicians to review emerging safety and pharmacokinetic data and to make key decisions about the trial. In particular, the safety review/dose-escalation committee will consult on advances in participant recruitment, when appropriate to advance dose escalation and when to terminate the study.

\section{Audit and inspection}

Monitoring will include centralised review of data collection and other study documents for protocol compliance, data accuracy and completeness. All study documents will be made available to the study Sponsor, and representatives of regulatory bodies for monitoring or audit purposes.

\section{Publication policy}

Results from the trial will be published in scientific peerreviewed journals and presented at national and international conferences. Any data published or presented will be anonymous to protect the privacy of participants and published according with the Australian National Data Service guidelines for publishing and sharing sensitive data.

Presentations and/or written summaries on the study outcomes will also be provided on invitation from interested patient advocacy and/or consumer groups, or individuals. All publications must receive written approval from the principal investigator, members of the research team and the study sponsor, Flinders University, prior to submission.

\section{Author affiliations}

${ }^{1}$ Anatomical Pathology, Flinders University, Bedford Park, South Australia, Australia

${ }^{2}$ School of Medicine, Flinders University, Bedford Park, South Australia, Australia

${ }^{3}$ Respiratory and Sleep Services, Flinders Medical Centre, Bedford Park, South

Australia, Australia

${ }^{4}$ Sign Path Pharma,Inc, Sandy, Utah, USA

${ }^{5}$ Medical Oncology, Flinders Medical Centre, Bedford Park, South Australia, Australia

Contributors AJH, SK, BD, ALF, SN, CSK and PS were involved in the conception, design, planning and conduct of the study. AJH, ALF and SK wrote the manuscript. $K G$ was involved in study planning and conduct. All authors were involved in the final approval of the manuscript. All authors agreed to be accountable for all aspects of this work.

Funding This study is funded by a Flinders Foundation Tour de Cure Support Grant (Award number AH03390), and the Doug Henderson Research Fund bequest (Award number not applicable). The test drug LipoCurcis supplied in-kind to the study investigators by Sign Path Pharma, USA.

Competing interests SK, AF, AJH, SN, CSK, BD, none declared. PS, is the Vice President and Chief Science Officer of Sign Path Pharma Inc, US.

Patient consent for publication Not required.

Provenance and peer review Not commissioned; externally peer reviewed.

Supplemental material This content has been supplied by the author(s). It has not been vetted by BMJ Publishing Group Limited (BMJ) and may not have been 
peer-reviewed. Any opinions or recommendations discussed are solely those of the author(s) and are not endorsed by BMJ. BMJ disclaims all liability and responsibility arising from any reliance placed on the content. Where the content includes any translated material, BMJ does not warrant the accuracy and reliability of the translations (including but not limited to local regulations, clinical guidelines, terminology, drug names and drug dosages), and is not responsible for any error and/or omissions arising from translation and adaptation or otherwise.

Open access This is an open access article distributed in accordance with the Creative Commons Attribution Non Commercial (CC BY-NC 4.0) license, which permits others to distribute, remix, adapt, build upon this work non-commercially, and license their derivative works on different terms, provided the original work is properly cited, appropriate credit is given, any changes made indicated, and the use is non-commercial. See: http://creativecommons.org/licenses/by-nc/4.0/.

\section{ORCID iDs}

Ashleigh Jean Hocking http://orcid.org/0000-0001-8311-8984

Alexandra L Farrall http://orcid.org/0000-0002-0909-8978

\section{REFERENCES}

1 Psallidas I, Kalomenidis I, Porcel JM, et al. Malignant pleural effusion: from bench to bedside. Eur Respir Rev 2016;25:189-98.

2 Zamboni MM, da Silva CT, Baretta R, et al. Important prognostic factors for survival in patients with malignant pleural effusion. BMC Pulm Med 2015;15:29.

3 Porcel JM, Gasol A, Bielsa S, et al. Clinical features and survival of lung cancer patients with pleural effusions. Respirology 2015;20:654-9.

4 Clive AO, Kahan BC, Hooper CE, et al. Predicting survival in malignant pleural effusion: development and validation of the LENT prognostic score. Thorax 2014;69:1098-104.

5 Davies HE, Mishra EK, Kahan BC, et al. Effect of an indwelling pleural catheter vs chest tube and talc pleurodesis for relieving dyspnea in patients with malignant pleural effusion: the TIME2 randomized controlled trial. JAMA 2012;307:2383-9.

6 Bibby AC, Dorn P, Psallidas I, et al. ERS/EACTS statement on the management of malignant pleural effusions. Eur $J$ Cardiothorac Surg 2019;55:116-32.

7 Feller-Kopman DJ, Reddy CB, DeCamp MM, et al. Management of malignant pleural effusions. An official ATS/STS/STR clinical practice guideline. Am J Respir Crit Care Med 2018;198:839-49.

8 Yeh H-H, Lai W-W, Chen HHW, et al. Autocrine IL-6-induced STAT3 activation contributes to the pathogenesis of lung adenocarcinoma and malignant pleural effusion. Oncogene 2006;25:4300-9.

9 Stathopoulos GT, Kollintza A, Moschos C, et al. Tumor necrosis factor-alpha promotes malignant pleural effusion. Cancer Res 2007;67:9825-34.

10 Shanmugam MK, Rane G, Kanchi MM, et al. The multifaceted role of curcumin in cancer prevention and treatment. Molecules 2015;20:2728-69.

11 Heger M, van Golen RF, Broekgaarden M, et al. The molecular basis for the pharmacokinetics and pharmacodynamics of curcumin and its metabolites in relation to cancer. Pharmacol Rev 2014;66:222-307.

12 Liu L, Sun L, Wu Q, et al. Curcumin loaded polymeric micelles inhibit breast tumor growth and spontaneous pulmonary metastasis. Int $J$ Pharm 2013;443:175-82.

13 Feng T, Wei Y, Lee RJ, et al. Liposomal curcumin and its application in cancer. Int J Nanomedicine 2017;12:6027-44.
14 Sercombe L, Veerati T, Moheimani F, et al. Advances and challenges of liposome assisted drug delivery. Front Pharmacol 2015;6:286.

15 Storka A, Vcelar B, Klickovic U, et al. Safety, tolerability and pharmacokinetics of liposomal curcumin in healthy humans. Int J Clin Pharmacol Ther 2015;53:54-65.

16 Greil R, Greil-Ressler S, Weiss L, et al. A phase 1 dose-escalation study on the safety, tolerability and activity of liposomal curcumin (Lipocurc $^{\mathrm{TM}}$ ) in patients with locally advanced or metastatic cancer. Cancer Chemother Pharmacol 2018;82:695-706.

17 Matabudul D, Pucaj K, Bolger G. Tissue distribution of (Lipocurc ${ }^{\text {TM }}$ ) liposomal curcumin and tetrahydrocurcumin following twoand eight-hour infusions in beagle dogs. Anticancer Res 2012;32:4359-64.

18 Lu C, Perez-Soler R, Piperdi B, et al. Phase II study of a liposomeentrapped cisplatin analog (L-NDDP) administered intrapleurally and pathologic response rates in patients with malignant pleural mesothelioma. J Clin Oncol 2005;23:3495-501.

19 Perez-Soler R, Shin DM, Siddik ZH. Phase I clinical and pharmacological study of liposome-entrapped NDDP administered intrapleurally in patients with malignant pleural effusions. Clin Cancer Res 1997:3:373-9.

20 Vatandoust S, Bright T, Roy AC, et al. Phase I open-label trial of intraperitoneal paclitaxel in combination with intravenous cisplatin and oral capecitabine in patients with advanced gastric cancer and peritoneal metastases (IPGP study): study protocol. BMJ Open 2019;9:e026732.

21 Delgado G, Potkul RK, Treat JA, et al. A phase I/II study of intraperitoneally administered doxorubicin entrapped in cardiolipin liposomes in patients with ovarian cancer. Am J Obstet Gynecol 1989;160:812-9. discussion 17-9.

22 Hocking A, Tommasi S, Sordillo P, et al. The safety and exploration of the pharmacokinetics of intrapleural liposomal curcumin. Int $J$ Nanomedicine 2020;15:943-52.

23 Chan A-W, Tetzlaff JM, Gøtzsche PC, et al. Spirit 2013 explanation and elaboration: guidance for protocols of clinical trials. BMJ 2013;346:e7586.

24 Aaronson NK, Ahmedzai S, Bergman B, et al. The European organization for research and treatment of cancer QLQ-C30: a quality-of-life instrument for use in international clinical trials in oncology. J Natl Cancer Inst 1993;85:365-76.

25 Cheng AL, Hsu CH, Lin JK. Phase I clinical trial of curcumin, a chemopreventive agent, in patients with high-risk or pre-malignant lesions. Anticancer Res 2001;21:2895-900.

26 Sharma RA, Euden SA, Platton SL, et al. Phase I clinical trial of oral curcumin: biomarkers of systemic activity and compliance. Clin Cancer Res 2004;10:6847-54

27 Carroll RE, Benya RV, Turgeon DK, et al. Phase IIA clinical trial of curcumin for the prevention of colorectal neoplasia. Cancer Prev Res 2011;4:354-64.

28 Ravindranath V, Chandrasekhara N. Absorption and tissue distribution of curcumin in rats. Toxicology 1980;16:259-65.

29 Wahlström B, Blennow G. A study on the fate of curcumin in the rat. Acta Pharmacol Toxicol 1978;43:86-92.

30 Le Tourneau C, Lee JJ, Siu LL. Dose escalation methods in phase I cancer clinical trials. J Natl Cancer Inst 2009;101:708-20.

31 Common terminology criteria for adverse events (CTCAE) v5.0. published online on the National cancer Insitute website at, 2018 Available: https://ctep.cancer.gov/protocolDevelopment/electronic_ applications/ctc.htm\#ctc_50

32 Australian code for the responsible conduct of research Camberra: National health and medical Research Council 2018. 